\title{
A Double Inequality for the Trigamma Function and Its Applications
}

\author{
Zhen-Hang Yang, ${ }^{1}$ Yu-Ming Chu, ${ }^{1}$ and Xiao-Jing Tao ${ }^{2}$ \\ ${ }^{1}$ School of Mathematics and Computation Sciences, Hunan City University, Yiyang 413000, China \\ ${ }^{2}$ College of Mathematics and Econometrics, Hunan University, Changsha 410082, China \\ Correspondence should be addressed to Yu-Ming Chu; chuyuming2005@126.com
}

Received 19 March 2014; Accepted 26 May 2014; Published 16 July 2014

Academic Editor: Wing-Sum Cheung

Copyright (c) 2014 Zhen-Hang Yang et al. This is an open access article distributed under the Creative Commons Attribution License, which permits unrestricted use, distribution, and reproduction in any medium, provided the original work is properly cited.

We prove that $p=1$ and $q=2$ are the best possible parameters in the interval $(0, \infty)$ such that the double inequality $\left(e^{p /(x+1)}-e^{-p / x}\right) / 2 p<\psi^{\prime}(x+1)<\left(e^{q /(x+1)}-e^{-q / x}\right) / 2 q$ holds for $x>0$. As applications, some new approximation algorithms for the circumference ratio $\pi$ and Catalan constant $G=\sum_{n=0}^{\infty}\left((-1)^{n} /(2 n+1)^{2}\right)$ are given. Here, $\psi^{\prime}$ is the trigamma function.

\section{Introduction}

For real and positive values of $x$, the classical Euler's gamma function $\Gamma$ and its logarithmic derivative $\psi$, the so-called psi function, are defined as

$$
\Gamma(x)=\int_{0}^{\infty} t^{x-1} e^{-t} d t, \quad \psi(x)=\frac{\Gamma^{\prime}(x)}{\Gamma(x)} .
$$

For extension of these functions to complex variables and for basic properties, see [1]. The derivatives $\psi^{\prime}, \psi^{\prime \prime}, \psi^{\prime \prime \prime}, \ldots$ are known as polygamma functions (see [2]). In particular, $\psi^{\prime}$ is called trigamma function.

Recently, the bounds for the trigamma function using exponential functions have attracted the attention of many researchers. For example, Elezovic et al. [3] proved that the inequality

$$
\psi^{\prime}(x)<e^{-\psi(x)}
$$

holds for all $x>0$. In [4, Theorem 2.7], Batir proved that $a^{*}=1 / 2$ and $b^{*}=\pi^{2} e^{-2 \gamma} / 6$ are the best possible constants such that the double inequality

$$
\left(x+a^{*}\right) e^{-2 \psi(x+1)}<\psi^{\prime}(x+1)<\left(x+b^{*}\right) e^{-2 \psi(x+1)}
$$

holds for all $x>0$, where $\gamma$ is Euler's constant. Batir [4] also showed that

$$
\begin{aligned}
\frac{1}{2} & \left(\frac{2}{x^{2}}-1+e^{2 /(x+1)}-e^{-2 \psi(x+1)}\right) \\
& <\psi^{\prime}(x+1) \\
& <\frac{1}{2}\left(\frac{2}{x^{2}}+1-e^{-2 / x}+e^{-2 \psi(x+1)}\right)
\end{aligned}
$$

for all $x>0$. In [5, (1.11)], Guo and Qi established that

$$
\frac{\vartheta e^{\vartheta / x}}{x^{2}\left(e^{9 / x}-1\right)}<\psi^{\prime}(x)<\frac{\theta e^{\theta / x}}{x^{2}\left(e^{\theta / x}-1\right)}
$$

if $x>0$ and $0<\vartheta \leq 1, \theta \geq 2$. They [6, Lemma 2] found a very simple upper bound for trigamma function in terms of exponential function as follows:

$$
\psi^{\prime}(x)<e^{1 / x}-1
$$

for all $x>0$. The inequality (6) was generalized in [7, Theorem 3.1], [8, Theorem 1.1], and [9, Theorem 1.1] to a complete monotonicity which reads that the difference $e^{1 / x}-$ $\psi^{\prime}(x)$ is completely monotonic on $(0, \infty)$. Many other new results involving the psi and trigamma functions can be found in the literature $[10,11]$. 
Suppose that $m \in(0, \infty)$ and $g$ and $g_{m}$ are the real functions defined on $(0, \infty), m=\lambda$ is said to be the best possible constant in $(0, \infty)$ such that the inequality $g(x)>$ $(<) g_{m}(x)$ holds for all $x>0$ if $g_{\lambda}(x) \geq(\leq) g_{\mu}(x)$ on $(0, \infty)$, or $g_{\lambda}(x)$ and $g_{\mu}(x)$ are not comparable on $(0, \infty)$, and $\lim _{x \rightarrow \infty}\left(g(x)-g_{\lambda}(x)\right) /\left(g(x)-g_{\mu}(x)\right)=0$ for any $\mu \in(0, \infty)$ satisfies $g(x)>(<) g_{\mu}(x)$ on the interval $(0, \infty)$.

The main purpose of this paper is to find the best possible constants $p, q \in(0, \infty)$ such that the double inequality

$$
\theta(x, p)<\psi^{\prime}(x+1)<\theta(x, q)
$$

or equivalently

$$
\frac{1}{x^{2}}+\theta(x, p)<\psi^{\prime}(x)<\frac{1}{x^{2}}+\theta(x, q)
$$

holds for all $x>0$, where

$$
\theta(x, m)=\frac{e^{m /(x+1)}-e^{-m / x}}{2 m}, \quad m>0 .
$$

Our main result is the following Theorem 1.

Theorem 1. $p=1$ and $q=2$ are the best possible constants in the interval $(0, \infty)$ such that the double inequality $(7)$ or $(8)$ holds for all $x>0$.

From Theorem 1, we clearly see the following.

Corollary 2. The double inequality

$$
\sinh \frac{1}{x+1}<\psi^{\prime}(x+1)<\frac{1}{2} \sinh \frac{2}{x}
$$

holds for all $x>0$.

\section{Lemmas}

Lemma 3. Let the function $\theta$ be defined on $(0, \infty)^{2}$ by (9). Then the function $\theta$ is strictly decreasing with respect to $m$ on $(0,1]$ and strictly increasing on $[3 / 2,+\infty)$.

Proof. It follows from (9) that

$$
\begin{aligned}
\frac{m^{2} x}{2(m+x)} e^{m / x} \frac{\partial \theta}{\partial m} \\
=\left(1-\frac{x(x-m+1)}{(x+1)(m+x)} \exp \left(\frac{m}{x}+\frac{m}{x+1}\right)\right) \\
=: h_{m}(x), \\
\frac{\partial h_{m}}{\partial x}=\frac{m^{2}}{x} \frac{\exp ((m / x)((2 x+1) /(x+1)))}{(x+1)^{3}(m+x)^{2}} \\
\quad \times\left((3-2 m) x^{2}+(3-2 m) x+(1-m)\right) .
\end{aligned}
$$

If $m \in(0,1]$, then $\partial h_{m} / \partial x>0$; that is, $h_{m}$ is strictly increasing with respect to $x>0$. Therefore,

$$
h_{m}(x)<\lim _{x \rightarrow \infty} h_{m}(x)=0,
$$

which implies that $\partial \theta / \partial m<0$.
If $m \geq 3 / 2$, then $\partial h_{m} / \partial x<0$; that is, $h_{m}$ is strictly decreasing with respect to $x>0$, which leads to the conclusion that

$$
h_{m}(x)>\lim _{x \rightarrow \infty} h_{m}(x)=0 .
$$

Lemma 4. Let the function $\theta$ be defined on $(0, \infty)^{2}$ by $(9)$ and

$$
F_{m}(x)=\psi^{\prime}(x+1)-\theta(x, m) .
$$

Then the equation

$$
F_{m}\left(0^{+}\right)=\psi^{\prime}(1)-\frac{e^{m}}{2 m}=0
$$

has two roots

$$
m_{1}=0.5023 \cdots, \quad m_{2}=1.7510 \cdots
$$

such that $F_{m}\left(0^{+}\right)>0$ for $m \in\left(m_{1}, m_{2}\right)$ and $F_{m}\left(0^{+}\right)<0$ for $m \in\left(0, m_{1}\right) \cup\left(m_{2}, \infty\right)$.

Proof. Differentiation yields

$$
\frac{d}{d m} F_{m}\left(0^{+}\right)=-\frac{1}{2 m^{2}} e^{m}(m-1),
$$

which reveals that the function $m \mapsto F_{m}\left(0^{+}\right)$is strictly increasing on $(0,1)$ and strictly decreasing on $(1, \infty)$. Therefore, Lemma 4 follows from the piecewise monotonicity of the function $m \mapsto F_{m}\left(0^{+}\right)$and the numerical computations results:

$$
\begin{gathered}
F_{0.5023}\left(0^{+}\right)=\frac{1}{6} \pi^{2}-\frac{e^{0.5023}}{1.0046}=-1.685 \cdots \times 10^{-5}<0, \\
F_{0.5024}\left(0^{+}\right)=\frac{1}{6} \pi^{2}-\frac{e^{0.5024}}{1.0048}=1.46 \cdots \times 10^{-4}>0, \\
F_{1.751}\left(0^{+}\right)=\frac{1}{6} \pi^{2}-\frac{e^{1.751}}{3.502}=5.68 \cdots \times 10^{-5}>0, \\
F_{1.7511}\left(0^{+}\right)=\frac{1}{6} \pi^{2}-\frac{e^{1.7511}}{3.5022}=-1.374 \cdots \times 10^{-5}<0 .
\end{gathered}
$$

Lemma 5. Let $m \geq 0, m_{1}=0.5023 \cdots$, and $m_{2}=1.7510 \cdots$ and let $F_{m}(x)$ be defined as in Lemma 4. Then the following statements are true:

(i) if the inequality $F_{m}(x) \geq 0$ holds for all $x>0$, then $m \in\left[1, m_{2}\right]$;

(ii) if the inequality $F_{m}(x) \leq 0$ holds for all $x>0$, then $m \in\left(0, m_{1}\right] \cup[2, \infty)$. 
Proof. It follows from the series formulas that

$$
\begin{gathered}
\psi^{\prime}(x)=\frac{1}{x}+\frac{1}{2 x^{2}}+\frac{1}{6 x^{3}}-\frac{1}{30 x^{5}}+\cdots, \\
e^{m /(x+1)}=1+\frac{m}{x+1}+\frac{1}{2}\left(\frac{m}{x+1}\right)^{2}+\frac{1}{6}\left(\frac{m}{x+1}\right)^{3} \\
+\frac{1}{24}\left(\frac{m}{x+1}\right)^{5}+\cdots, \\
e^{-m / x}=1-\frac{m}{x}+\frac{1}{2}\left(-\frac{m}{x}\right)^{2}+\frac{1}{6}\left(-\frac{m}{x}\right)^{3}+\frac{1}{24}\left(-\frac{m}{x}\right)^{5}+\cdots,
\end{gathered}
$$

and we get

$$
\begin{gathered}
\lim _{x \rightarrow \infty} \frac{F_{m}(x)}{x^{-3}} \\
=\lim _{x \rightarrow \infty}\left(\left(\frac{1}{(x+1)}+\frac{1}{2(x+1)^{2}}+\frac{1}{6(x+1)^{3}}\right.\right. \\
-\left(\left(1+\frac{m}{x+1}+\frac{1}{2}\left(\frac{m}{x+1}\right)^{2}+\frac{1}{6}\left(\frac{m}{x+1}\right)^{3}\right.\right. \\
\left.-\left(1-\frac{m}{x}+\frac{1}{2}\left(-\frac{m}{x}\right)^{2}+\frac{1}{6}\left(-\frac{m}{x}\right)^{3}\right)\right) \\
\left.\times\left(x^{-3}\right)^{-1}\right) \\
=-\frac{1}{12} \lim _{x \rightarrow \infty}\left(\left(\left(2 m^{2}-6 m+4\right) x^{3}+\left(3 m^{2}-9 m+6\right) x^{2}\right.\right. \\
\left.+\left(3 m^{2}-3 m\right) x+m^{2}\right) \\
\left.\times\left((x+1)^{3}\right)^{-1}\right) \\
=-\frac{1}{12}\left(2 m^{2}-6 m+4\right)=-\frac{1}{6}(m-1)(m-2) .
\end{gathered}
$$

(i) If inequality $F_{m}(x) \geq 0$ holds for all $x>0$, then, from

$$
\begin{aligned}
& F_{m}\left(0^{+}\right)=\psi^{\prime}(1)-\frac{e^{m}}{2 m} \geq 0, \\
& \lim _{x \rightarrow \infty} \frac{F_{m}(x)}{x^{-3}}=-\frac{1}{6}(m-1)(m-2) \geq 0
\end{aligned}
$$

and Lemma 4 , we clearly see that $m \in\left[m_{1}, m_{2}\right] \cap[1,2]=$ $\left[1, m_{2}\right]$.

(ii) If inequality $F_{m}(x) \leq 0$ holds for all $x>0$, then

$$
\begin{aligned}
& F_{m}\left(0^{+}\right)=\psi^{\prime}(1)-\frac{e^{m}}{2 m} \leq 0, \\
& \lim _{x \rightarrow \infty} \frac{F_{m}(x)}{x^{-3}}=-\frac{1}{6}(m-1)(m-2) \leq 0
\end{aligned}
$$

and Lemma 4 lead to the conclusion that

$$
\begin{aligned}
m & \in\left(\left(0, m_{1}\right] \cup\left[m_{2}, \infty\right]\right) \cap((0,1] \cup[2, \infty)) \\
& =\left(0, m_{1}\right] \cup[2, \infty) .
\end{aligned}
$$

Lemma 6. Let the function $\theta$ be defined on $(0, \infty)^{2}$ by (9). Then $\theta(x, 1)$ and $\theta(x, m)$ are not comparable for all $x>0$ if $m \in(1,2)$.

Proof. For $x>0$ and $m>0$, let

$$
\begin{aligned}
G_{1, m}(x)= & \theta(x, 1)-\theta(x, m) \\
= & \frac{e^{1 /(x+1)}-e^{-1 / x}}{2} \\
& -\frac{e^{m /(x+1)}-e^{-m / x}}{2 m} .
\end{aligned}
$$

Then simple computation leads to

$$
G_{1, m}\left(0^{+}\right)=\frac{e}{2}-\frac{e^{m}}{2 m} .
$$

From (20) and (21), we have

$$
\lim _{x \rightarrow \infty} \frac{G_{1, m}(x)}{x^{-3}}=-\frac{1}{6}(m-1)(m-2) .
$$

Differentiation yields

$$
\frac{d}{d m} G_{1, m}\left(0^{+}\right)=-\frac{e^{m}}{2 m^{2}}(m-1),
$$

which shows that $m \mapsto G_{1, m}\left(0^{+}\right)$is strictly decreasing on $(1, \infty)$. Therefore,

$$
G_{1, m}\left(0^{+}\right)<G_{1,1}\left(0^{+}\right)=0
$$

if $m \in(1,2)$. On the other hand, we clearly see that $\lim _{x \rightarrow \infty}\left[x^{3} G_{1, m}(x)\right]>0$ for $m \in(1,2)$.

Lemma 7. Let $n \in \mathbb{N}=\{1,2,3, \ldots\}$ and $m \in \mathbb{N} \cup\{0\}$ with $n>m$ and let $P_{n}(t)$ be the polynomial of degree $n$ defined by

$$
P_{n}(t)=\sum_{i=m+1}^{n} a_{i} t^{i}-\sum_{i=0}^{m} a_{i} t^{i},
$$

where $a_{n}, a_{m}>0$ and $a_{i} \geq 0$ for $0 \leq i \leq n-1$ with $i \neq m$. Then, there exists $t_{m+1} \in(0, \infty)$ such that $P_{n}\left(t_{m+1}\right)=0$ and $P_{n}(t)<0$ for $t \in\left(0, t_{m+1}\right)$ and $P_{n}(t)>0$ for $t \in\left(t_{m+1}, \infty\right)$.

Proof. Differentiating $P_{n}(t)$ gives

$$
\begin{aligned}
P_{n}^{(k)}(t)= & \sum_{i=m+1}^{n} \frac{i !}{(i-k) !} a_{i} t^{i-k} \\
& -\sum_{i=k}^{m} \frac{i !}{(i-k) !} a_{i} t^{i-k} \quad \text { for } 1 \leq k \leq m, \\
P_{n}^{(m+1)}(t) & =\sum_{i=m+1}^{n} \frac{i !}{(i-m-1) !} a_{i} t^{i-m-1}>0 .
\end{aligned}
$$


Note that

$$
P_{n}^{(k)}(\infty)=\infty, \quad P_{n}^{(k)}\left(0^{+}\right)=-a_{k}
$$

for $1 \leq k \leq m$.

From $P_{n}^{(m+1)}(t)>0$, we clearly see that $P_{n}^{(m)}(t)$ is strictly increasing on $(0, \infty)$. Then $P_{n}^{(m)}(\infty)>0$ and $P_{n}^{(m)}\left(0^{+}\right)=$ $-a_{m}<0$ lead to the conclusion that there exists $t_{1} \in(0, \infty)$ such that $P_{n}^{(m)}\left(t_{1}\right)=0$ and $P_{n}^{(m)}(t)<0$ for $t \in\left(0, t_{1}\right)$ and $P_{n}^{(m)}(t)>0$ for $t \in\left(t_{1}, \infty\right)$. Therefore, $P_{n}^{(m-1)}(t)$ is strictly decreasing on $\left(0, t_{1}\right)$ and strictly increasing on $\left(t_{1}, \infty\right)$.

It follows from the piecewise monotonicity of $P_{n}^{(m-1)}(t)$ and $P_{n}^{(m-1)}(\infty)=\infty$ that $P_{n}^{(m-1)}(t)<P_{n}^{(m-1)}\left(0^{+}\right)=-a_{m-1} \leq$ 0 for $t \in\left(0, t_{1}\right)$, and there exists $t_{2} \in\left(t_{1}, \infty\right)$ such that $P_{n}^{(m-1)}\left(t_{2}\right)=0$ and $P_{n}^{(m-1)}(t)<0$ for $t \in\left(0, t_{2}\right)$ and $P_{n}^{(m-1)}(t)>$ 0 for $t \in\left(t_{2}, \infty\right)$. Therefore, $P_{n}^{(m-2)}(t)$ is strictly decreasing on $\left(0, t_{2}\right)$ and strictly increasing on $\left(t_{2}, \infty\right)$.

After repeating the same steps as above $m+1$ times, we deduce that there exists $t_{m+1} \in\left(t_{m}, \infty\right) \subset(0, \infty)$ such that $P_{n}\left(t_{m+1}\right)=0$ and $P_{n}(t)<0$ for $t \in\left(0, t_{m+1}\right)$ and $P_{n}(t)>0$ for $t \in\left(t_{m+1}, \infty\right)$.

Lemma 8. Let the function $\theta$ be defined on $(0, \infty)^{2}$ by (9). Then there exists $m_{0} \in(2 / 5,9 / 20)$ such that $\theta(x, 2)$ and $\theta(x, m)$ are not comparable for all $x>0$ if $m \in\left(m_{0}, 1\right)$, and $\theta(x, 2)<\theta(x, m)$ for all $x>0$ if $m \in\left(0, m_{0}\right]$.

Proof. For $x>0$ and $m>0$, let

$$
\begin{aligned}
G_{2, m}(x)= & \theta(x, 2)-\theta(x, m) \\
= & \frac{e^{2 /(x+1)}-e^{-2 / x}}{4} \\
& -\frac{e^{m /(x+1)}-e^{-m / x}}{2 m} .
\end{aligned}
$$

Then simple computation leads to

$$
G_{2, m}\left(0^{+}\right)=\frac{e^{2}}{4}-\frac{e^{m}}{2 m} .
$$

(i) We prove that there exists $m_{0} \in(2 / 5,9 / 20)$ such that $\theta(x, 2)$ and $\theta(x, m)$ are not comparable for all $x>0$ if $m \in$ $\left(m_{0}, 1\right)$. For this end, it suffices to prove that there exists $m_{0} \in$ $(2 / 5,9 / 20)$ such that $G_{2, m}\left(0^{+}\right)>0$ and $\lim _{x \rightarrow \infty} x^{3} G_{2, m}(x)<$ 0 if $m \in\left(m_{0}, 1\right)$.

Indeed, it follows from

$$
\frac{d}{d m} G_{2, m}\left(0^{+}\right)=-\frac{e^{m}}{2 m^{2}}(m-1)
$$

that the function $m \mapsto G_{2, m}\left(0^{+}\right)$is strictly increasing on $(0,1)$. Numerical computations show that

$$
\begin{aligned}
& G_{2,2 / 5}\left(0^{+}\right)=\frac{1}{4} e^{2}-\frac{5}{4} e^{2 / 5}<0, \\
& G_{2,9 / 20}\left(0^{+}\right)=\frac{1}{4} e^{2}-\frac{10}{9} e^{9 / 20}>0 .
\end{aligned}
$$

Therefore, there exists $m_{0} \in(2 / 5,9 / 20)$ such that

$$
G_{2, m}\left(0^{+}\right)=\frac{e^{2}}{4}-\frac{e^{m}}{2 m}=0
$$

and $G_{2, m}\left(0^{+}\right)<0$ for $m \in\left(0, m_{0}\right)$ and $G_{2, m}\left(0^{+}\right)>0$ for $m \in$ $\left(m_{0}, 1\right)$.

On the other hand, it follows from $m \in\left(m_{0}, 1\right)$ together with (20) and (21) that

$$
\lim _{x \rightarrow \infty} \frac{G_{2, m}(x)}{x^{-3}}=-\frac{1}{6}(m-1)(m-2)<0 .
$$

(ii) We prove that $\theta(x, 2)<\theta(x, m)$ for all $x>0$ if $m \in\left(0, m_{0}\right]$. From Lemma 3 , we know that $m \mapsto \theta(x, m)$ is strictly decreasing on $(0,1]$, so it suffices to prove that $\theta(x, 2)<\theta\left(x, m_{0}\right)$ for all $x>0$.

Let

$$
\begin{aligned}
g_{1}(x)= & e^{m_{0} / x} G_{2, m_{0}}(x) \\
= & \frac{1}{4} \exp \left(\frac{m_{0}}{x}+\frac{2}{x+1}\right)-\frac{1}{4} \exp \left(\frac{m_{0}}{x}-\frac{2}{x}\right) \\
& +\frac{1}{2 m_{0}}\left(1-\exp \left(\frac{m_{0}}{x}+\frac{m_{0}}{x+1}\right)\right) .
\end{aligned}
$$

Then

$$
\lim _{x \rightarrow \infty} g_{1}(x)=0 .
$$

Differentiation yields

$$
\begin{aligned}
& \frac{4}{2-m_{0}} x^{2} e^{\left(2-m_{0}\right) / x} \times g_{1}^{\prime}(x) \\
& =\frac{2\left(2 x^{2}+2 x+1\right)}{\left(2-m_{0}\right)(x+1)^{2}} \exp \left(\frac{2}{x}+\frac{m_{0}}{x+1}\right)-1 \\
& \quad-\frac{\left(m_{0}+2\right) x^{2}+2 m_{0} x+m_{0}}{\left(2-m_{0}\right)(x+1)^{2}} \exp \left(\frac{2}{x}+\frac{2}{x+1}\right) \\
& :=g_{2}(x),
\end{aligned}
$$

$$
\begin{aligned}
g_{2}^{\prime}(x)= & 2\left(\left(2 m_{0}+2\right) x^{4}+\left(2 m_{0}+10\right) x^{3}\right. \\
& \left.+\left(m_{0}+14\right) x^{2}+8 x+2\right) \\
& \times\left(\left(2-m_{0}\right) x^{2}(x+1)^{4}\right)^{-1} \\
& \times \exp \left(\frac{m_{0}}{x+1}+\frac{2}{x}\right) g_{3}(x),
\end{aligned}
$$

where

$$
\begin{aligned}
g_{3}(x)= & \left(\left(2 m_{0}+2\right) x^{4}+\left(6 m_{0}+2\right) x^{3}\right. \\
& \left.+\left(7 m_{0}+2\right) x^{2}+4 m_{0} x+m_{0}\right) \\
& \times\left(\left(2 m_{0}+2\right) x^{4}+\left(2 m_{0}+10\right) x^{3}\right. \\
& \left.\quad+\left(m_{0}+14\right) x^{2}+8 x+2\right)^{-1} \\
& \times e^{\left(2-m_{0}\right) /(x+1)}-1 .
\end{aligned}
$$


Note that

$$
\begin{gathered}
\lim _{x \rightarrow \infty} g_{2}(x)=0, \\
\lim _{x \rightarrow 0^{+}} g_{3}(x)=\frac{1}{2} m_{0} e^{2-m_{0}}-1=0, \\
\lim _{x \rightarrow \infty} g_{3}(x)=0,
\end{gathered}
$$

where the second equation in (46) follows from (38).

Differentiating $g_{3}(x)$ leads to

$$
\begin{aligned}
g_{3}^{\prime}(x)= & \left(\left(2-m_{0}\right) e^{\left(2-m_{0}\right) /(x+1)}\right) \\
& \times\left(( x + 1 ) ^ { 2 } \left(8 x+m_{0} x^{2}+2 m_{0} x^{3}\right.\right. \\
& \left.\left.\quad+2 m_{0} x^{4}+14 x^{2}+10 x^{3}+2 x^{4}+2\right)^{2}\right)^{-1} \\
& \times g_{4}(x),
\end{aligned}
$$

where

$$
\begin{aligned}
g_{4}(x)= & \left(4-4 m_{0}^{2}\right) x^{8}+\left(16-16 m_{0}^{2}\right) x^{7} \\
& +\left(-28 m_{0}^{2}-24 m_{0}+32\right) x^{6} \\
& +\left(-28 m_{0}^{2}-72 m_{0}+40\right) x^{5} \\
& -\left(17 m_{0}^{2}+104 m_{0}-42\right) x^{4} \\
& -\left(6 m_{0}^{2}+88 m_{0}-36\right) x^{3} \\
& -\left(m_{0}^{2}+46 m_{0}-18\right) x^{2} \\
& -\left(14 m_{0}-4\right) x-2 m_{0} \\
:= & a_{8} x^{8}+a_{7} x^{7}+a_{6} x^{6}+a_{5} x^{5}-a_{4} x^{4} \\
& -a_{3} x^{3}-a_{2} x^{2}-a_{1} x-a_{0} .
\end{aligned}
$$

We assert that there exists a unique $x_{4}^{*} \in(0, \infty)$ such that $g_{4}(x)<0$ for $x \in\left(0, x_{4}^{*}\right)$ and $g_{4}(x)>0$ for $x \in\left(x_{4}^{*}, \infty\right)$, which leads to the conclusion that $g_{3}(x)$ is strictly decreasing on $\left(0, x_{4}^{*}\right]$ and strictly increasing on $\left[x_{4}^{*}, \infty\right)$. To this end, it is enough to verify that the coefficients of $g_{4}(x)$ satisfy the conditions of Lemma 7. In fact, it follows from $m_{0} \in$ $(2 / 5,9 / 20):=\left(m_{0-}, m_{0+}\right)$ that we have

$$
\begin{aligned}
a_{8} & =4-4 m_{0}^{2}=4\left(1-m_{0}^{2}\right)>0, \\
a_{7} & =16-16 m_{0}^{2}=16\left(1-m_{0}^{2}\right)>0, \\
a_{6} & =-28 m_{0}^{2}-24 m_{0}+32 \\
& >-28 m_{0+}^{2}-24 m_{0+}+32=\frac{1553}{100}>0,
\end{aligned}
$$

$$
\begin{aligned}
a_{5} & =-28 m_{0}^{2}-72 m_{0}+40 \\
& >-28 m_{0+}^{2}-72 m_{0+}+40=\frac{193}{100}>0, \\
a_{4} & =\left(17 m_{0}^{2}+104 m_{0}-42\right) \\
& >17 m_{0-}^{2}+104 m_{0-}-42=\frac{58}{25}>0, \\
a_{3} & =\left(6 m_{0}^{2}+88 m_{0}-36\right) \\
& >6 m_{0-}^{2}+88 m_{0-}-36=\frac{4}{25}>0, \\
a_{2} & =\left(m_{0}^{2}+46 m_{0}-18\right) \\
& >m_{0-}^{2}+46 m_{0-}-18=\frac{14}{25}>0, \\
a_{1} & =\left(14 m_{0}-4\right) \\
& >14 m_{0-}-4=\frac{8}{5}>0, \\
a_{0} & =2 m_{0}>0 .
\end{aligned}
$$

From the piecewise monotonicity of $g_{3}(x)$ together with (46) and (47), we clearly see that

$$
\begin{array}{ll}
g_{3}(x)<\lim _{x \rightarrow 0^{+}} g_{3}(x)=0 & \text { for } x \in\left(0, x_{4}^{*}\right], \\
g_{3}(x)<\lim _{x \rightarrow \infty} g_{3}(x)=0 & \text { for } x \in\left[x_{4}^{*}, \infty\right) ;
\end{array}
$$

that is, $g_{3}(x)<0$ for $x \in(0, \infty)$. Then (43) and (45) lead to the conclusion that $g_{2}(x)>\lim _{x \rightarrow \infty} g_{2}(x)=0$ for $x \in(0, \infty)$, which implies that $g_{1}(x)$ is strictly increasing on $(0, \infty)$ and $g_{1}(x)<\lim _{x \rightarrow \infty} g_{1}(x)=0$ for $x \in(0, \infty)$.

Therefore, $\theta(x, 2)<\theta\left(x, m_{0}\right)$ follows easily from (40) and $g_{1}(x)<0$.

Lemma 9 (see [12, pp. 258-260]). Let $x>0$ and $n \in$ $\{0,1,2, \ldots\}$. Then

$$
\psi^{(n)}(x+1)-\psi^{(n)}(x)=\frac{(-1)^{n} n !}{x^{n+1}} .
$$

From the proof of [4, Theorem 2.6], we get the following.

Lemma 10. The inequality

$$
\psi^{\prime}(x+1)>\frac{2 x+1}{2 x^{2}+2 x+2 / 3}
$$

holds for $x>-1$.

The following lemma can be derived immediately from the proof of [4, Theorem 2.1].

Lemma 11 (see [4, Theorem 2.1]). Let $y$ be the function defined on $(0, \infty)$ by

$$
y(x)=e^{2 \psi(x)} .
$$

Then $y^{\prime \prime \prime}(x)>0$ for $x \in(0, \infty)$. 
The well-known Hermite-Hadamard inequality for convex function can be stated as follows.

Lemma 12 (see [13]). Let $I \subseteq \mathbb{R}$ be an interval, $a, b \in I$ with $a<b$, and let $f: I \rightarrow \mathbb{R}$ be a convex function. Then

$$
f\left(\frac{a+b}{2}\right) \leq \frac{\int_{a}^{b} f(x) d x}{b-a} \leq \frac{f(a)+f(b)}{2} .
$$

\section{Proofs of Theorem 1}

Proof of Theorem 1. We divide the proof into four parts.

(I) We prove the first inequality in (7); that is,

$$
F_{1}(x)=\psi^{\prime}(x+1)-\frac{e^{1 /(x+1)}-e^{-1 / x}}{2}>0,
$$

where $F_{m}(x)$ is defined by (14).

It follows from Lemma 10 that

$$
\begin{aligned}
F_{1}(x) & =\psi^{\prime}(x+1)-\frac{e^{1 /(x+1)}-e^{-1 / x}}{2} \\
& >\frac{2 x+1}{2 x^{2}+2 x+2 / 3}-\frac{e^{1 /(x+1)}-e^{-1 / x}}{2} \\
& :=e^{-1 / x} H(x)
\end{aligned}
$$

where

$$
\begin{aligned}
H(x)= & \frac{2 x+1}{2 x^{2}+2 x+2 / 3} e^{1 / x} \\
& -\frac{\exp ((1 /(x+1))+(1 / x))-1}{2} .
\end{aligned}
$$

We clearly see that it is enough to prove that $H(x)>0$ for $x>0$.

Differentiating $H(x)$ gives

$$
\begin{aligned}
H^{\prime}(x)= & \frac{1}{2 x^{2}} e^{1 / x} \\
& \times\left(\frac{2 x^{2}+2 x+1}{(x+1)^{2}} e^{1 /(x+1)}\right. \\
= & \left.\frac{1}{2 x^{2}} e^{1 / x} L \quad \frac{3(x+1)\left(6 x^{3}+6 x^{2}+4 x+1\right)}{\left(3 x^{2}+3 x+1\right)^{2}}\right) \\
& \times\left(\frac{2 x^{2}+2 x+1}{(x+1)^{2}} e^{1 /(x+1)},\right. \\
& \left.\frac{3(x+1)\left(6 x^{3}+6 x^{2}+4 x+1\right)}{\left(3 x^{2}+3 x+1\right)^{2}}\right) \times h(x),
\end{aligned}
$$

where $L(a, b)=(b-a) /(\ln a-\ln b)$ denotes the logarithmic mean of positive numbers $a$ and $b$, and

$$
\begin{aligned}
h(x)= & \frac{1}{x+1}+\ln \frac{2 x^{2}+2 x+1}{(x+1)^{2}} \\
& -\ln \frac{3(x+1)\left(6 x^{3}+6 x^{2}+4 x+1\right)}{\left(3 x^{2}+3 x+1\right)^{2}}
\end{aligned}
$$

Differentiating $h(x)$ leads to

$$
\begin{aligned}
h^{\prime}(x)= & \left(x(2 x+1)\left(7 x^{2}+7 x+3\right)\right) \\
& \times\left((x+1)^{2}\left(3 x^{2}+3 x+1\right)\left(2 x^{2}+2 x+1\right)\right. \\
& \left.\times\left(6 x^{3}+6 x^{2}+4 x+1\right)\right)^{-1}>0
\end{aligned}
$$

for $x>0$, which means that $h$ is strictly increasing on $(0, \infty)$ and $h(x)<\lim _{x \rightarrow \infty} h(x)=0$. It in turn implies that $H$ is strictly decreasing on $(0, \infty)$. Therefore, $H(x)>$ $\lim _{x \rightarrow \infty} H(x)=0$ for $x>0$.

(II) We prove that $m=1$ is the best possible constant such that $\psi^{\prime}(x+1)>\theta(x, m)$ for all $x>0$.

From Lemma 5, we know that $m \in\left[1, m_{2}\right]$ if $\psi^{\prime}(x+1)>$ $\theta(x, m)$ for all $x>0$, where $m_{2}=1.7510 \ldots$. It follows from Lemma 6 that $\theta(x, 1)$ and $\theta(x, m)$ are not comparable for all $x>0$ if $m \in(1,2)$; that is to say, $\theta\left(x, m^{*}\right)$ is not a better lower bound of $\psi^{\prime}(x+1)$ than $\theta(x, 1)$ even if there exists $m^{*} \epsilon$ $\left(1, m_{2}\right]$ such that $\psi^{\prime}(x+1)>\theta\left(x, m^{*}\right)$.

For any $m^{*} \in\left(1, m_{2}\right],(22)$ leads to

$$
\lim _{x \rightarrow \infty}\left[x^{3} F_{m^{*}}(x)\right]=\frac{1}{6}\left(m^{*}-1\right)\left(2-m^{*}\right) .
$$

It follows from (19), (20), and (21) that we get

$$
\lim _{x \rightarrow \infty}\left[x^{5} F_{1}(x)\right]=\frac{1}{24}
$$

(III) We prove the second inequality (7); that is,

$$
F_{2}(x)=\psi^{\prime}(x+1)-\frac{e^{2 /(x+1)}-e^{-2 / x}}{4}<0
$$

for $x>0$, where $F_{m}(x)$ is defined by (14).

Lemma 11 implies that the function $x \mapsto y^{\prime}(x)=$ $2 \psi^{\prime}(x) e^{2 \psi(x)}$ is strictly convex on $(0, \infty)$. Then, making use of Lemma 12, we get

$$
y^{\prime}\left(\frac{x+x+2}{2}\right)<\frac{\int_{x}^{x+2} y^{\prime}(t) d t}{x+2-x}
$$

for $x>0$. That is,

$$
\begin{gathered}
2 \psi^{\prime}(x+1) e^{2 \psi(x+1)}<\frac{e^{2 \psi(x+2)}-e^{2 \psi(x)}}{2}, \\
\psi^{\prime}(x+1)<\frac{1}{4}\left[e^{2(\psi(x+2)-\psi(x+1))}-e^{2(\psi(x)-\psi(x+1))}\right] .
\end{gathered}
$$


Therefore, inequality (64) follows easily from (52) and the last inequality above.

(IV) We prove that $m=2$ is the best possible constant such that $\psi^{\prime}(x+1)<\theta(x, m)$ for all $x>0$.

From Lemma 5, we know that $m \in\left(0, m_{1}\right] \cup[2, \infty)$ if $\psi^{\prime}(x+1)<\theta(x, m)$ for all $x>0$, where $m_{1}=0.5023 \cdots$.

It follows from Lemma 8 that there exists $m_{0} \in$ $(2 / 5,9 / 20) \subset\left(0, m_{1}\right)$ such that $\theta(x, 2)$ and $\theta(x, m)$ are not comparable for all $x>0$ if $m \in\left(m_{0}, m_{1}\right]$ and $\theta(x, 2)<\theta(x, m)$ for all $x>0$ if $m \in\left(0, m_{0}\right]$. Lemma 3 leads to the conclusion that $\theta(x, 2)<\theta(x, m)$ for all $x>0$ if $m \in(2, \infty)$.

If there exists $m^{*} \in\left(m_{0}, m_{1}\right]$ such that $F_{m^{*}}(x)=\psi^{\prime}(x+$ 1) $-\theta\left(x, m^{*}\right)<0$ for all $x>0$, then, from (19), (20), (21), and (22), we get

$$
\begin{gathered}
\lim _{x \rightarrow \infty}\left[x^{3} F_{m^{*}}(x)\right]=-\frac{1}{6}\left(1-m^{*}\right)\left(2-m^{*}\right), \\
\lim _{x \rightarrow \infty}\left[x^{7} F_{2}(x)\right]=-\frac{1}{45} .
\end{gathered}
$$

From the above proof and Lemma 3 we get the following.

Corollary 13. Let the function $\theta$ be defined on $(0, \infty)^{2}$ by (9) and let $m_{0} \in(2 / 5,9 / 20)$ be the root of $(38)$ on $(0,1)$. Then the inequalities

$$
\begin{aligned}
\psi^{\prime}(x+1) & <\theta(x, 2)<\theta\left(x, m_{0}\right)<\lim _{m \rightarrow 0} \theta(x, m) \\
& =\frac{1}{2(x+1)}+\frac{1}{2 x}
\end{aligned}
$$

or equivalently

$$
\psi^{\prime}(x)<\frac{1}{2(x+1)}+\frac{1}{2 x}+\frac{1}{x^{2}}
$$

hold for all $x>0$.

\section{Remarks}

Remark 14. It follows from (67) and the facts that

$$
\begin{aligned}
& \lim _{x \rightarrow \infty} \frac{\psi^{\prime}(x)-e^{-\psi(x)}}{x^{-3}}=-\frac{1}{24}, \\
& \lim _{x \rightarrow \infty} \frac{\psi^{\prime}(x+1)-\left(x+\pi^{2} e^{-2 \gamma} / 6\right) e^{-2 \psi(x+1)}}{x^{-2}} \\
& =\frac{1}{2}-\frac{1}{6} \pi^{2} e^{-2 \gamma}, \\
& \lim _{x \rightarrow \infty} \frac{\psi^{\prime}(x+1)-(1 / 2)\left(\left(2 / x^{2}\right)+1-e^{-2 / x}+e^{-2 \psi(x+1)}\right)}{x^{-6}} \\
& =-\frac{1}{60}, \\
& \lim _{x \rightarrow \infty} \frac{\psi^{\prime}(x)-\left(e^{1 / x}-1\right)}{x^{-4}}=-\frac{1}{24}, \\
& \lim _{x \rightarrow \infty} \frac{\psi^{\prime}(x)-\left(2 e^{2 / x} / x^{2}\left(e^{2 / x}-1\right)\right)}{x^{-2}}=-\frac{1}{2}
\end{aligned}
$$

that we clearly see that the upper bound in Theorem 1 for the trigamma function $\psi^{\prime}$ is better than the upper bounds given in (2), (3), (4), (5), and (6) if $x$ is large enough.

Lemma 15. One has

$$
\begin{aligned}
& e^{1 /(x+1)}+e^{-1 / x}>2 \quad \text { if } x \in(-\infty,-1) \cup(0, \infty), \\
& e^{1 /(x+1)}+e^{-1 / x} \geq 2 e^{2} \quad \text { if } x \in(-1,0) .
\end{aligned}
$$

Proof. Differentiation leads to

$$
\begin{aligned}
& \left(e^{1 /(x+1)}+e^{-1 / x}\right)^{\prime} \\
& =\frac{e^{-1 / x}}{x^{2}}\left(1-\frac{x^{2}}{(x+1)^{2}} \exp \left(\frac{1}{x+1}+\frac{1}{x}\right)\right) \\
& \quad:=\frac{e^{-1 / x}}{x^{2}} V(x), \\
& V^{\prime}(x)=\frac{\exp (1 /(x+1)+(1 / x))}{(x+1)^{4}}>0 .
\end{aligned}
$$

If $x \in(0, \infty)$, then, from $V^{\prime}(x)>0$, we get $V(x)<$ $\lim _{x \rightarrow \infty} V(x)=0$, which leads to

$$
e^{1 /(x+1)}+e^{-1 / x}>\lim _{x \rightarrow \infty}\left(e^{1 /(x+1)}+e^{-1 / x}\right)=2 .
$$

If $x \in(-\infty,-1)$, then the first inequality in (71) still holds by making a change of variable $y=-(x+1)$. If $x \in(-1,0)$, since $V(-1 / 2)=0$, we see that $V(x)<V(-1 / 2)=0$ for $x \in(-1,-1 / 2)$ and $V(x)>V(-1 / 2)=0$ for $x \in(-1 / 2,0)$. Hence,

$$
e^{1 /(x+1)}+e^{-1 / x} \geq\left[e^{1 /(x+1)}+e^{-1 / x}\right]_{x=-1 / 2}=2 e^{2}
$$

Remark 16. Using inequality (71), one has

$$
\begin{aligned}
\frac{1}{x^{2}} & +\theta(x, 2)-\left(e^{1 / x}-1\right) \\
& =\frac{1}{x^{2}}+\frac{e^{2 /(x+1)}-e^{-2 / x}}{4}-\left(e^{1 / x}-1\right) \\
& <\frac{1}{x^{2}}+\frac{e^{2 /(x+1)}-\left(2-e^{1 /(x+1)}\right)^{2}}{4}-\left(e^{1 / x}-1\right) \\
& =\frac{1}{x^{2}}+e^{1 /(x+1)}-e^{1 / x}=\frac{1}{x^{2}}+\sum_{n=0}^{\infty} \frac{1}{n !}\left(\frac{1}{(x+1)^{n}}-\frac{1}{x^{n}}\right) \\
& <\frac{1}{x^{2}}+\sum_{n=0}^{3} \frac{1}{n !}\left(\frac{1}{(x+1)^{n}}-\frac{1}{x^{n}}\right)=-\frac{1}{6 x^{3}(x+1)^{3}}<0
\end{aligned}
$$

for $x>0$, which shows that the upper bound $x^{-2}+\theta(x, 2)$ in $(8)$ is better than the upper bound $\left(e^{1 / x}-1\right)$ in (6). 
Remark 17. The conclusion that the difference $e^{1 / x}-\psi^{\prime}(x)$ is completely monotonic on $(0, \infty)$ given in $[6$, Lemma 2$]$ implies that

$$
e-\psi^{\prime}(1)>e^{1 /(x+1)}-\psi^{\prime}(x+1)>1
$$

or

$$
e^{1 /(x+1)}-e+\frac{\pi^{2}}{6}<\psi^{\prime}(x+1)<e^{1 /(x+1)}-1<\frac{1}{2} \sinh \frac{2}{x} .
$$

It is easy to check that the lower bound $e^{1 /(x+1)}-e+\pi^{2} / 6$ and $\sinh 1 /(x+1)$ for $\psi^{\prime}(x+1)$ given in (10) are not comparable due to

$$
e^{1 /(x+1)}-e+\frac{\pi^{2}}{6}>(<) \sinh \frac{1}{x+1} \quad \text { if } x<(>) 1.62670 \cdots .
$$

Remark 18. Guo et al. [14] proved that

$$
\frac{(k-1) !}{x^{k}}+\frac{k !}{2 x^{k+1}}<(-1)^{k+1} \psi^{(k)}(x)<\frac{(k-1) !}{x^{k}}+\frac{k !}{x^{k+1}}
$$

for $x>0$ if $k \in \mathbb{N}$. In particular, if $k=1$, one has

$$
\frac{1}{x}+\frac{1}{2 x^{2}}<\psi^{\prime}(x)<\frac{1}{x}+\frac{1}{x^{2}}
$$

We clearly see that the upper bound given in (69) is better than that in (80) for the trigamma function $\psi^{\prime}(x)$.

Finally, we give remarks on two mathematical constants $\pi$ and $G$ (Catalan constant).

Remark 19. It is well known that

$$
\sum_{k=1}^{\infty} \frac{1}{k^{2}}=\frac{\pi^{2}}{6}
$$

Let $Q_{n}=\sum_{k=1}^{n}\left(1 / k^{2}\right)$, and then $\psi^{\prime}(n+1)=\left(\pi^{2} / 6\right)-Q_{n}$. From Theorem 1, we clearly see that the double inequality

$$
\frac{e^{1 /(n+1)}-e^{-1 / n}}{2}<\frac{\pi^{2}}{6}-Q_{n}<\frac{e^{2 /(n+1)}-e^{-2 / n}}{4}
$$

holds for all $n \in \mathbb{N}$.

Let

$$
l_{n}=Q_{n}+\frac{e^{1 /(n+1)}-e^{-1 / n}}{2}, \quad L_{n}=Q_{n}+\frac{e^{2 /(n+1)}-e^{-2 / n}}{4} \text {. }
$$

Then inequalities (82) can be rewritten as

$$
l_{n}<\frac{\pi^{2}}{6}<L_{n}
$$

or

$$
\sqrt{6 l_{n}}<\pi<\sqrt{6 L_{n}}
$$

TABle 1

\begin{tabular}{lccc}
\hline$n$ & $\left|\sqrt{6 Q_{n}}-\pi\right|$ & $\left|\sqrt{6 l_{n}}-\pi\right|$ & $\left|\sqrt{6 L_{n}}-\pi\right|$ \\
\hline 1 & 0.6921 & $4.3127 \times 10^{-3}$ & $7.663 \times 10^{-4}$ \\
2 & 0.40298 & $3.7549 \times 10^{-4}$ & $2.8250 \times 10^{-5}$ \\
5 & 0.1782 & $7.7596 \times 10^{-6}$ & $1.3276 \times 10^{-7}$ \\
10 & $9.2231 \times 10^{-2}$ & $3.1013 \times 10^{-7}$ & $1.4875 \times 10^{-9}$ \\
50 & $1.8966 \times 10^{-2}$ & $1.2112 \times 10^{-10}$ & $2.5320 \times 10^{-14}$ \\
100 & $9.5161 \times 10^{-3}$ & $3.8807 \times 10^{-12}$ & $2.0489 \times 10^{-16}$ \\
200 & $4.7663 \times 10^{-3}$ & $1.2280 \times 10^{-13}$ & $1.6291 \times 10^{-18}$ \\
500 & $1.9085 \times 10^{-3}$ & $1.2669 \times 10^{-15}$ & $2.6973 \times 10^{-21}$ \\
\hline
\end{tabular}

It follows from (63) and (67) that

$$
\begin{aligned}
& \lim _{n \rightarrow \infty} n^{5}\left(l_{n}-\frac{\pi^{2}}{6}\right)=-\frac{1}{24}, \\
& \lim _{n \rightarrow \infty} n^{7}\left(L_{n}-\frac{\pi^{2}}{6}\right)=\frac{1}{45} .
\end{aligned}
$$

Therefore, (85) provides a new approximation algorithm for $\pi$. Numerical simulations results carried out with mathematical software show that the given algorithm is more accurate than $\sqrt{6 Q_{n}}$ (see Table 1$)$.

More estimate methods for $\pi$ can be found in [15-19].

Remark 20. The Catalan constant

$$
G=\sum_{n=0}^{\infty} \frac{(-1)^{n}}{(2 n+1)^{2}}=0.9159655941772190 \cdots
$$

is a mysterious constant in mathematics and physics. From $\psi^{\prime}(1 / 4)=\pi^{2}+8 G$ and $\psi^{\prime}(n+(1 / 4)+1)=\psi^{\prime}(1 / 4)-\sum_{k=0}^{n}(1 /(k+$ $\left.1 / 4)^{2}\right)($ see $[17,20])$ together with Theorem 1 , we get

$$
\begin{array}{r}
\frac{e^{1 /(n+5 / 4)}-e^{-1 /(n+1 / 4)}}{16}+\frac{1}{8} \sum_{k=0}^{n} \frac{1}{(k+1 / 4)^{2}}-\frac{\pi^{2}}{8}<G \\
<\frac{e^{2 /(n+5 / 4)}-e^{-2 /(n+1 / 4)}}{32}+\frac{1}{8} \sum_{k=0}^{n} \frac{1}{(k+1 / 4)^{2}}-\frac{\pi^{2}}{8}
\end{array}
$$

for $n \in \mathbb{N}$.

Let

$$
\begin{aligned}
& u_{n}=\frac{e^{1 /(n+5 / 4)}-e^{-1 /(n+1 / 4)}}{16}+\frac{1}{8} \sum_{k=0}^{n} \frac{1}{(k+1 / 4)^{2}}-\frac{\pi^{2}}{8}, \\
& U_{n}=\frac{e^{2 /(n+5 / 4)}-e^{-2 /(n+1 / 4)}}{32}+\frac{1}{8} \sum_{k=0}^{n} \frac{1}{(k+1 / 4)^{2}}-\frac{\pi^{2}}{8} .
\end{aligned}
$$

Then inequalities (88) can be rewritten as

$$
u_{n}<G<U_{n} .
$$

It follows from (63) and (67) that we have

$$
\begin{aligned}
& \lim _{n \rightarrow \infty} n^{5}\left(u_{n}-G\right)=-\frac{1}{24}, \\
& \lim _{n \rightarrow \infty} n^{7}\left(U_{n}-G\right)=\frac{1}{45} .
\end{aligned}
$$


TABLE 2

\begin{tabular}{lccc}
\hline$n$ & $\left|\sum_{k=0}^{n}(-1)^{k} /(2 k+1)^{2}-G\right|$ & $\left|u_{n}-G\right|$ & $\left|U_{n}-G\right|$ \\
\hline 1 & $2.7077 \times 10^{-2}$ & $2.7274 \times 10^{-4}$ & $3.7885 \times 10^{-5}$ \\
2 & $1.2923 \times 10^{-2}$ & $3.0912 \times 10^{-5}$ & $1.9591 \times 10^{-6}$ \\
5 & $3.4037 \times 10^{-3}$ & $8.1456 \times 10^{-7}$ & $1.2782 \times 10^{-8}$ \\
10 & $1.0268 \times 10^{-3}$ & $3.6098 \times 10^{-8}$ & $1.6524 \times 10^{-10}$ \\
50 & $4.8045 \times 10^{-5}$ & $1.5468 \times 10^{-11}$ & $3.2017 \times 10^{-15}$ \\
100 & $1.2253 \times 10^{-5}$ & $5.0171 \times 10^{-13}$ & $2.6358 \times 10^{-17}$ \\
200 & $3.0939 \times 10^{-6}$ & $1.5974 \times 10^{-14}$ & $2.1139 \times 10^{-19}$ \\
500 & $4.98 \times 10^{-7}$ & $1.6542 \times 10^{-16}$ & $3.5184 \times 10^{-22}$ \\
\hline
\end{tabular}

Therefore, (90) provides a new approximation algorithm for the Catalan constant $G$. Numerical simulations results carried out with mathematical software show that the given algorithm is more accurate than $\sum_{k=0}^{n}\left((-1)^{k} /(2 k+1)^{2}\right)$ (see Table 2).

\section{Conflict of Interests}

The authors declare that there is no conflict of interests regarding the publication of this paper.

\section{Acknowledgments}

This research was supported by the Natural Science Foundation of China under Grants 61374086 and 11171307 and the Natural Science Foundation of Zhejiang Province under Grant LY13A010004.

\section{References}

[1] E. T. Whittaker and G. N. Watson, A Course of Modern Analysis, Cambridge University Press, New York, NY, USA, 1962.

[2] G. D. Anderson and S.-L. Qiu, "A monotoneity property of the gamma function," Proceedings of the American Mathematical Society, vol. 125, no. 11, pp. 3355-3362, 1997.

[3] N. Elezović, C. Giordano, and J. Pečarić, "The best bounds in Gautschi's inequality," Mathematical Inequalities \& Applications, vol. 3, no. 2, pp. 239-252, 2000.

[4] N. Batir, "Sharp bounds for the psi function and harmonic numbers," Mathematical Inequalities \& Applications, vol. 14, no. 4, pp. 917-925, 2011.

[5] B.-N. Guo and F. Qi, "Some properties of the psi and polygamma functions," Hacettepe Journal of Mathematics and Statistics, vol. 39, no. 2, pp. 219-231, 2010.

[6] B.-N. Guo and F. Qi, "Refinements of lower bounds for polygamma functions," Proceedings of the American Mathematical Society, vol. 141, no. 3, pp. 1007-1015, 2013.

[7] F. Qi and C. Berg, "Complete monotonicity of a difference between the exponential and trigamma functions and properties related to a modified Bessel function," Mediterranean Journal of Mathematics, vol. 10, no. 4, pp. 1685-1696, 2013.

[8] F. Qi and S.-H. Wang, "Complete monotonicity, completely monotonic degree, integral representations, and an inequality related to the exponential, trigamma, and modified Bessel functions," http://arxiv.org/abs/1210.2012.
[9] F. Qi and X.-J. Zhang, "Complete monotonicity of a difference between the exponential and trigamma functions," http://arxiv.org/abs/1203.1582.

[10] F. Qi, " Properties of modified Bessel functions and completely monotonic degrees of differences between exponential and trigamma functions," http://arxiv.org/abs/1302.6731.

[11] W. Li, F. Qi, and B. Guo, "On proofs for monotonicity of a function involving the psi and exponential functions," Analysis. International Mathematical Journal of Analysis and its Applications, vol. 33, no. 1, pp. 44-50, 2013.

[12] M. Abramowitz and I. A. Stegun, Handbook of Mathematical Functions with Formulas, Graphs, and Mathematical Tables, Dover, New York, NY, USA, 1972.

[13] J. E. Pečarić, F. Proschan, and Y.-L. Tong, Convex Functions, Partial Orderings, and Statistical Applications, Academic Press, Boston, Mass, USA, 1992.

[14] B. N. Guo, R. J. Chen, and F. Qi, "A class of completely monotonic functions involving the polygamma functions," Journal of Mathematical Analysis and Approximation Theory, vol. 1, no. 2, pp. 124-134, 2006.

[15] D. Bailey, P. Borwein, and S. Plouffe, "On the rapid computation of various polylogarithmic constants," Mathematics of Computation, vol. 66, no. 218, pp. 903-913, 1997.

[16] J. M. Borwein, P. B. Borwein, and K. Dilcher, "Pi, Euler numbers, and asymptotic expansions," American Mathematical Monthly, vol. 96, no. 8, pp. 681-687, 1989.

[17] D. Borwein, J. M. Borwein, M. L. Glasser, and J. Wan, “Moments of Ramanujan's generalized elliptic integrals and extensions of Catalan's constant," Journal of Mathematical Analysis and Applications, vol. 384, no. 2, pp. 478-496, 2011.

[18] R. C. Gupta, "On the remainder term in the Mādhava-Leibniz's series," Ganita Bhäratī, vol. 14, no. 1-4, pp. 68-71, 1992.

[19] S. Ramanujan, "Modular equations and approximations to $\pi$," Quarterly Journal of Mathematics, vol. 45, pp. 350-372, 1914.

[20] K. S. Kölbig, "The polygamma function $\psi^{(k)}(x)$ for $x=1 / 4$ and $x=3 / 4$," Journal of Computational and Applied Mathematics, vol. 75, no. 1, pp. 43-46, 1996. 


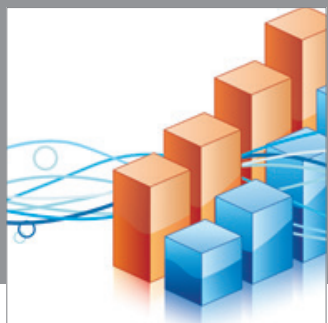

Advances in

Operations Research

mansans

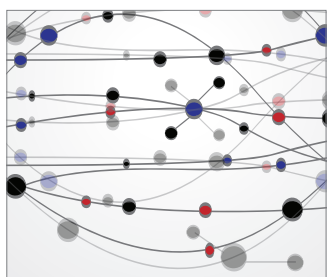

The Scientific World Journal
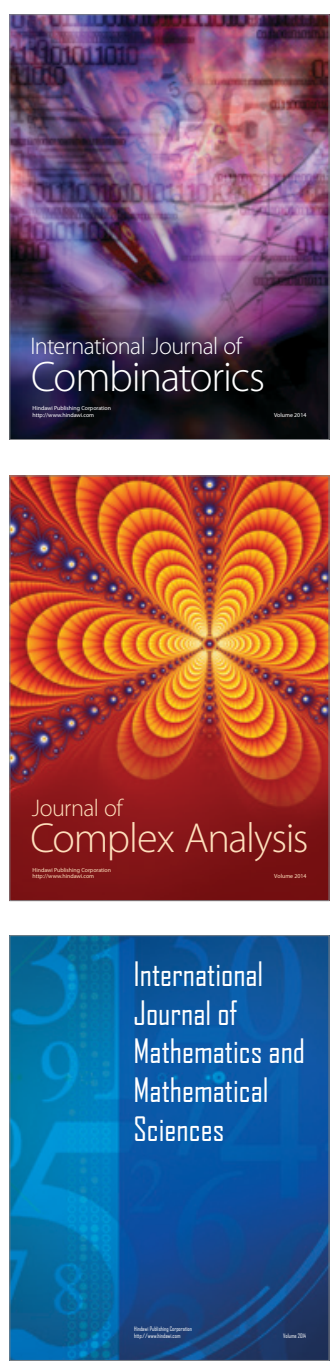
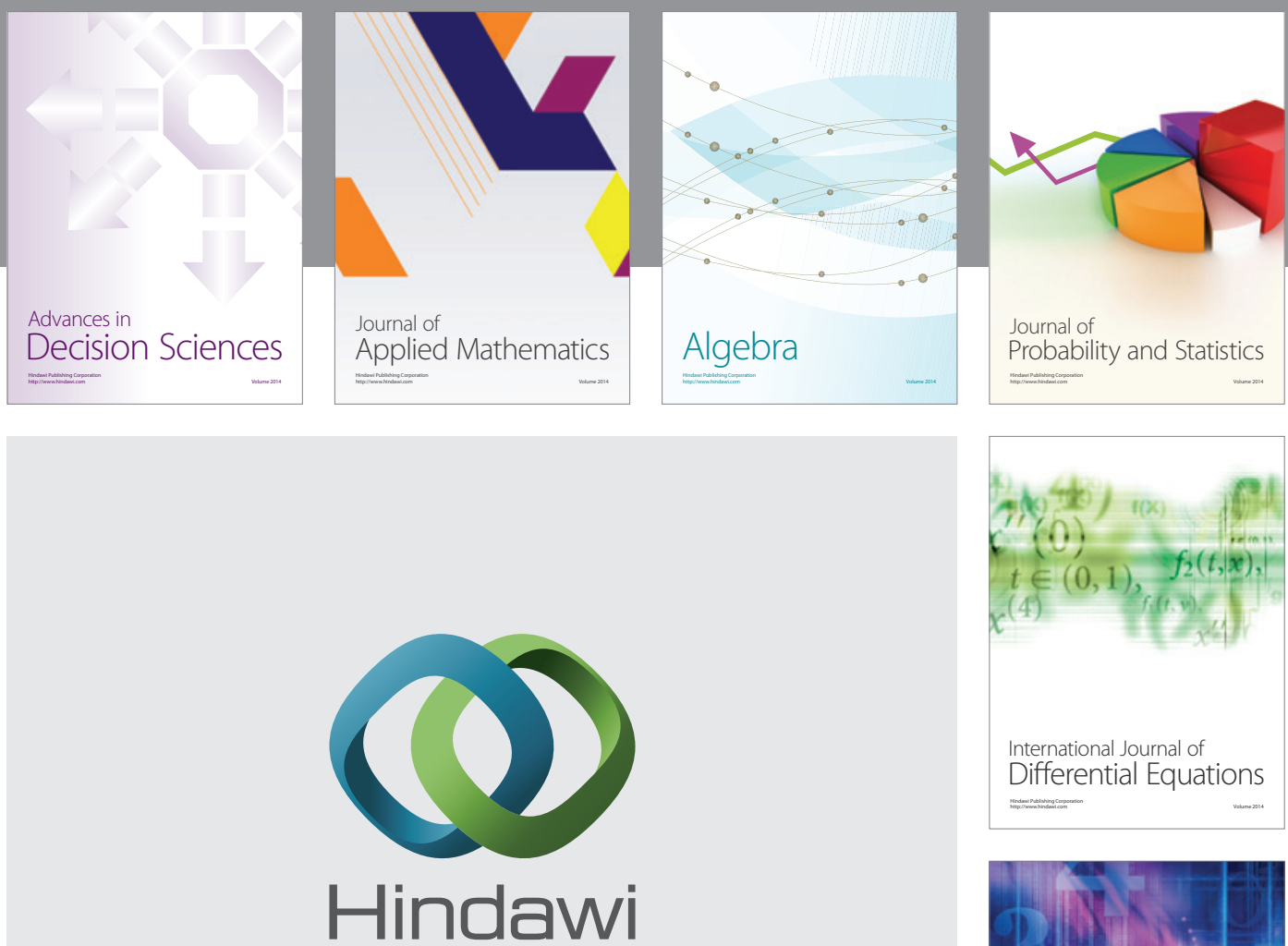

Submit your manuscripts at http://www.hindawi.com
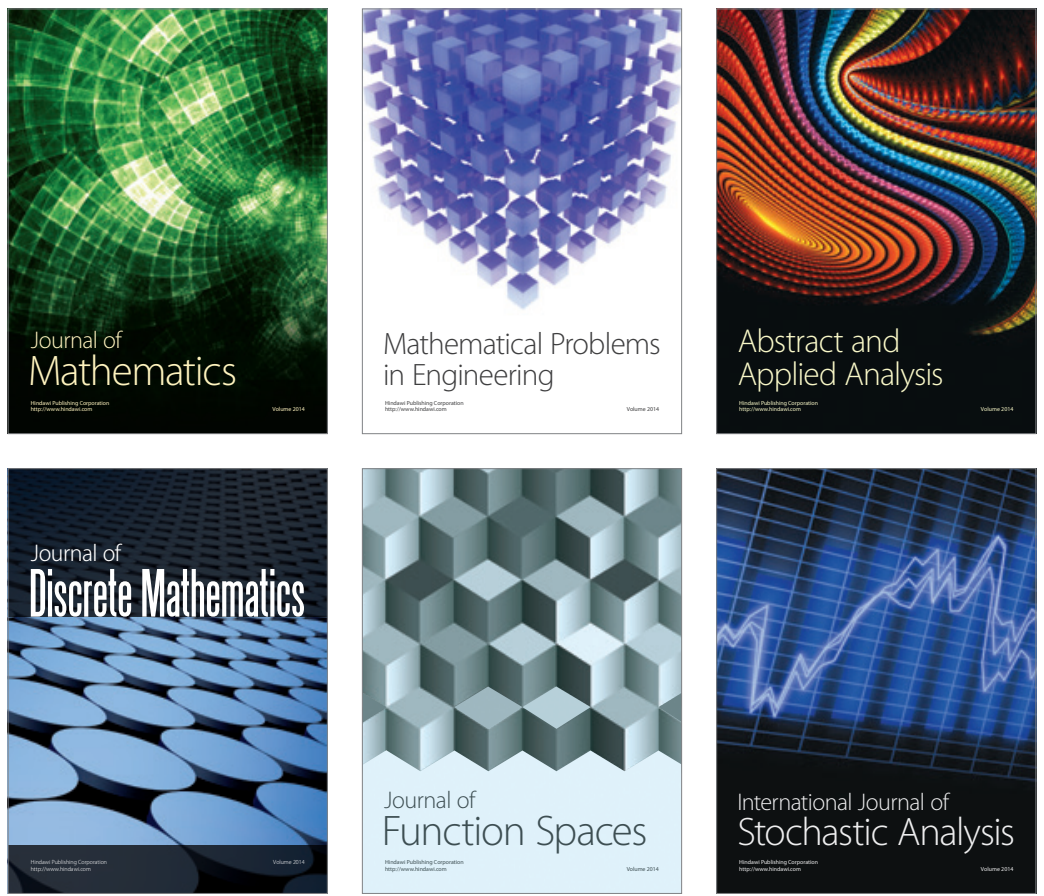

Journal of

Function Spaces

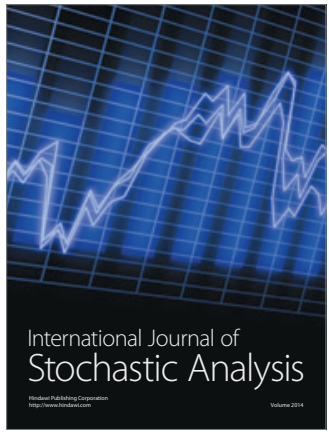

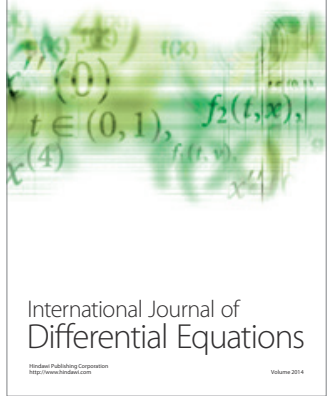
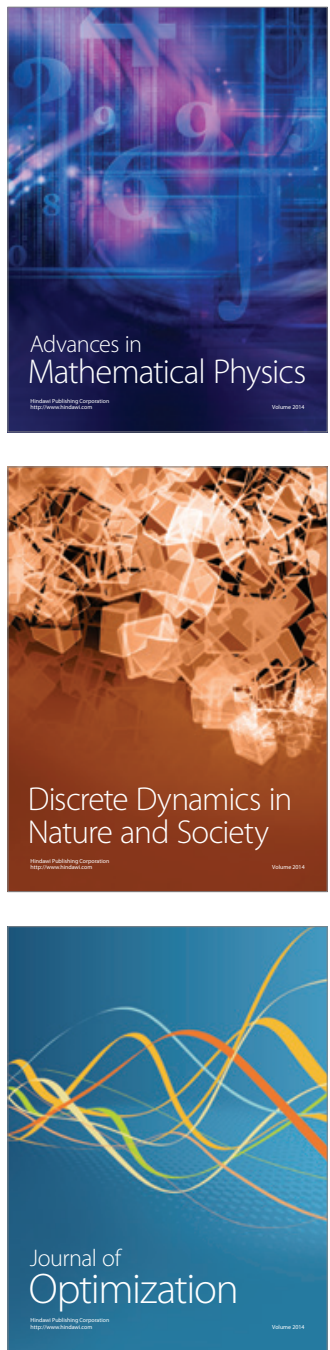